\title{
On Paranormed Ideal Convergent Generalized Difference Strongly Summable Sequence Spaces Defined over $\boldsymbol{n}$-Normed Spaces
}

\author{
Bipan Hazarika \\ Department of Mathematics, Rajiv Gandhi University, Rono Hills, Doimukh, Itanagar-791 112, \\ Arunachal Pradesh, India \\ Correspondence should be addressed to Bipan Hazarika, bh_rgu@yahoo.co.in
}

Received 25 January 2011; Accepted 3 April 2011

Academic Editor: G. Ólafsson

Copyright (C 2011 Bipan Hazarika. This is an open access article distributed under the Creative Commons Attribution License, which permits unrestricted use, distribution, and reproduction in any medium, provided the original work is properly cited.

\begin{abstract}
We introduce a new class of ideal convergent (shortly I-convergent) sequence spaces using an Orlicz function and difference operator of order $s \geq 1$ defined over the $n$-normed spaces. We investigate these spaces for some linear topological structures. These investigations will enhance the acceptability of the notion of $n$-norm by giving a way to construct different sequence spaces with elements in $n$-normed spaces. We also give some relations related to these sequence spaces.
\end{abstract}

\section{Introduction}

Throughout the paper $w, \ell_{\infty}, c, c_{0}$, and $\ell_{p}$ denote the classes of all, bounded, convergent, null, and $p$-absolutely summable sequences of complex numbers.

The notion of statistical convergence is a very useful functional tool for studying the convergence problems of numerical sequences/matrices (double sequences) through the concept of density. It was first introduced by Fast [1], and Schoenberg [2], independently for the real sequences. Later on, it was further investigated from sequence point of view and linked with the summability theory by Fridy [3] and many others. The idea is based on the notion of natural density of subsets of $\mathbb{N}$, the set of positive integers, which is defined as follows: the natural density of a subset $E$ of $\mathbb{N}$ is denoted by $\delta(E)$ and is defined by

$$
\delta(E)=\lim _{n \rightarrow \infty} \frac{1}{n}|\{k \in E: k \leq n\}|,
$$

where the vertical bar denotes the cardinality of the respective set. 
The notion of $I$-convergence ( $I$ denotes the ideal of subsets of $\mathbb{N}$ ), which is a generalization of statistical convergence, was introduced by Kastyrko et al. [4] and further studied by many other authors.

The notion of difference sequence space was introduced by Kizmaz [5], who studied the difference sequence spaces $\ell_{\infty}(\Delta), c(\Delta)$, and $c_{0}(\Delta)$. The notion was further generalized by Et and Çolak [6] by introducing the sequence spaces $\ell_{\infty}\left(\Delta^{s}\right), c\left(\Delta^{s}\right)$, and $c_{0}\left(\Delta^{s}\right)$. Another type of generalization of the difference sequence spaces is due to Tripathy and Esi [7], who studied the spaces $\ell_{\infty}\left(\Delta_{m}\right), c\left(\Delta_{m}\right)$, and $c_{0}\left(\Delta_{m}\right)$. Tripathy et al. [8] generalized the above notion and unified these as follows.

For nonnegative integers $m, s$, the generalized difference sequence spaces are defined as follows: for a given sequence space $Z$, we have

$$
Z\left(\Delta_{m}^{s}\right)=\left\{x=\left(x_{k}\right) \in w:\left(\Delta_{m}^{s} x_{k}\right) \in Z\right\}
$$

where $\Delta_{m}^{s} x=\left(\Delta_{m}^{s} x_{k}\right)=\left(\Delta_{m}^{s-1} x_{k}-\Delta_{m}^{s-1} x_{k+m}\right), \Delta_{m}^{0} x_{k}=x_{k}$, for all $k \in \mathbb{N}$, the difference operator is equivalent to the following binomial representation:

$$
\Delta_{m}^{s} x_{k}=\sum_{v=0}^{s}(-1)^{v}\left(\begin{array}{l}
s \\
v
\end{array}\right) x_{k+m v}
$$

Taking $s=1$, we get the spaces $\ell_{\infty}\left(\Delta_{m}\right), c\left(\Delta_{m}\right)$, and $c_{0}\left(\Delta_{m}\right)$, introduced and studied by Tripathy and Et [7]. Taking $m=1$, we get the spaces $\ell_{\infty}\left(\Delta^{s}\right), c\left(\Delta^{s}\right)$, and $c_{0}\left(\Delta^{s}\right)$ studied by Et and Çolak [6]. Taking $s=m=1$, we get the spaces $\ell_{\infty}(\Delta), c(\Delta)$, and $c_{0}(\Delta)$ introduced and studied by Kizmaz [5].

Let $m, s$ be nonnegative integers, then for a given sequence space $Z$, we introduced

$$
Z\left(\Delta_{m}^{(s)}\right)=\left\{x=\left(x_{k}\right) \in w:\left(\Delta_{m}^{(s)} x_{k}\right) \in Z\right\}
$$

where $\Delta_{\mathrm{m}}^{(\mathrm{s})} x=\left(\Delta_{\mathrm{m}}^{(\mathrm{s})} x_{k}\right)=\left(\Delta_{\mathrm{m}}^{(\mathrm{s}-1)} x_{k}-\Delta_{\mathrm{m}}^{(\mathrm{s}-1)} x_{k-m}\right), \Delta_{\mathrm{m}}^{(0)} x_{k}=x_{k}$, for all $k \in \mathbb{N}$, the difference operator is equivalent to the following binomial representation:

$$
\Delta_{m}^{(s)} x_{k}=\sum_{v=0}^{s}(-1)^{v}\left(\begin{array}{l}
s \\
v
\end{array}\right) x_{k-m v},
$$

where $x_{k}=0$, for $k<0$.

The concept of 2-normed space was initially introduced by Gähler [9], in the mid of 1960s, while that of $n$-normed spaces can be found in Misiak [10]. Since then, many others authors have used this concept and obtained various results, see, for instance, Gunawan [11] and Gunawan and Mashadi [12,13]. Recently, a lot of activities have started to study summability, sequence spaces, and related topics in these spaces (see [14,15]).

The notion of ideal-convergence in 2-normed spaces was initially introduced by Gürdal [16]. Later on, it was extended to $n$-normed spaces by Gürdal and Sahnier [17].

An Orlicz function is a function $M:[0, \infty) \rightarrow[0, \infty)$, which is continuous, nondecreasing and convex with $M(0)=0, M(0)>0$, as $x>0$ and $M(x) \rightarrow \infty$, as $x \rightarrow \infty$. 
An Orlicz function $M$ can always be represented in the following integral form:

$$
M(x)=\int_{0}^{x} p(t) d t
$$

where $p$ is the known kernel of $M$, right differentiable for $t \geq 0, p(0)=0, p(t)>0$, for $t>0$ and $p(t) \rightarrow \infty$, as $t \rightarrow \infty$.

If convexity of Orlicz function $M$ is replaced by $M(x+y) \leq M(x)+M(y)$, then this function is called Modulus function, which was presented and discussed by Maddox [18].

Lindenstrauss and Tzafriri [19] used the idea of Orlicz function to construct the sequence space

$$
\ell_{M}=\left\{\left(x_{k}\right) \in w: \sum_{k=1}^{\infty} M\left(\frac{\left|x_{k}\right|}{\rho}\right)<\infty, \text { for some } \rho>0\right\} .
$$

The space $\ell_{M}$ with the norm

$$
\|x\|=\inf \left\{\rho>0: \sum_{k=1}^{\infty} M\left(\frac{\left|x_{k}\right|}{\rho}\right) \leq 1\right\}
$$

becomes a Banach space which is called an Orlicz sequence space.

The space $\ell_{M}$ is closely related to the space $\ell_{p}$ which is an Orlicz sequence space with $M(t)=|t|^{p}$, for $1 \leq p<\infty$.

Subsequently, Orlicz function was used to define sequence spaces by Parashar and Choudhary [20] and others (see [10, 15, 21-23]).

Remark 1.1. It is well known if $M$ is a convex function and $M(0)=0$, then

$$
M(\lambda x) \leq \lambda M(x), \quad \forall \lambda \text { with } 0<\lambda<1 .
$$

In this paper, we introduce some new ideal convergent sequences over $n$-normed spaces by using Orlicz functions and generalized difference sequence spaces.

\section{Definitions and Preliminaries}

Let $n$ be a nonnegative integer and $X$ a real vector space of dimension $d \geq n$ ( $d$ may be infinite). A real-valued function $\|\cdot, \ldots, \cdot\|$ on $X^{n}$ satisfying the following conditions:

(1) $\left\|x_{1}, x_{2}, \ldots, x_{n}\right\|=0$ if and only if $x_{1}, x_{2}, \ldots, x_{n}$ are linearly dependent,

(2) $\left\|x_{1}, x_{2}, \ldots, x_{n}\right\|$ is invariant under permutation,

(3) $\left\|\alpha x_{1}, x_{2}, \ldots, x_{n}\right\|=|\alpha|\left\|x_{1}, x_{2}, \ldots, x_{n}\right\|$, for any $\alpha \in \mathbb{R}$,

(4) $\left\|x+x^{\prime}, x_{2}, \ldots, x_{n}\right\| \leq\left\|x, x_{2}, \ldots, x_{n}\right\|+\left\|x^{\prime}, x_{2}, \ldots, x_{n}\right\|$,

is called an $n$-norm on $X$ and the pair $(X,\|., \ldots,\|$.$) is called an n$-normed space. 
A trivial example of an $n$-normed space is $X=\mathbb{R}^{n}$, equipped with the Euclidean $n$ norm $\left\|x_{1}, x_{2}, \ldots, x_{n}\right\|_{E}=$ the volume of the $n$-dimensional parallelopiped spanned by the vectors $x_{1}, x_{2}, \ldots, x_{n}$, which may be given explicitly by the formula

$$
\left\|x_{1}, x_{2}, \ldots, x_{n}\right\|_{E}=\left|\operatorname{det}\left(x_{i j}\right)\right|=\operatorname{abs}\left(\left|\begin{array}{ccc}
\left\langle x_{1}, x_{1}\right\rangle & \cdots & \left\langle x_{1}, x_{n}\right\rangle \\
\cdot & \cdots & \cdot \\
\cdot & \cdots & . \\
\left\langle x_{n}, x_{1}\right\rangle & \cdots & \left\langle x_{n}, x_{n}\right\rangle
\end{array}\right|\right) \text {, }
$$

where $x_{i}=\left(x_{i 1}, x_{i 2}, \ldots, x_{i n}\right) \in \mathbb{R}^{n}$, for each $i=1,2, \ldots, n$.

Let $(X,\|., \ldots,\|$.$) be an n$-normed space of dimension $d \geq n \geq 2$ and $\left\{a_{1}, a_{2}, \ldots, a_{n}\right\}$ a linearly independent set in $X$. Then, the function $\|\cdot, \ldots, \cdot\|_{\infty}$ on $X^{n-1}$ is defined by

$$
\left\|x_{1}, x_{2}, \ldots, x_{n}\right\|_{\infty}=\max _{1 \leq i \leq n}\left\{\left\|x_{1}, x_{2}, \ldots, x_{n-1}, a_{i}\right\|\right\}
$$

defines as $(n-1)$-norm on $X$ with respect to $\left\{a_{1}, a_{2}, \ldots, a_{n}\right\}$, and this is known as the derived $(n-1)$-norm (see [12] for details).

The standard $n$-norm on $X$, a real inner product space of dimension $d \geq n$, is as follows:

$$
\left\|x_{1}, x_{2}, \ldots, x_{n}\right\|_{S}=\left|\begin{array}{ccc}
\left\langle x_{1}, x_{1}\right\rangle & \cdots & \left\langle x_{1}, x_{n}\right\rangle \\
\cdot & \cdots & \cdot \\
\cdot & \cdots & \cdot \\
\left\langle x_{n}, x_{1}\right\rangle & \cdots & \left\langle x_{n}, x_{n}\right\rangle
\end{array}\right|^{1 / 2},
$$

where $\langle\cdot, \cdot\rangle$ denotes the inner product on $X$. If we take $X=\mathbb{R}^{n}$, then this $n$-norm is exactly the same as the Euclidean $n$-norm $\left\|x_{1}, x_{2}, \ldots, x_{n}\right\|_{E}$ mentioned earlier.

For $n=1$, this $n$-norm is the usual norm $\left\|x_{1}\right\|=\sqrt{\left\langle x_{1}, x_{1}\right\rangle}$.

Definition 2.1. A sequence $\left(x_{k}\right)$ in an $n$-normed space $(X,\|\cdot, \ldots, \cdot\|)$ is said to converge to some $l \in X$ with respect the $n$-norm if for each $\varepsilon>0$, there exists an positive integer $n_{0}$ such that

$$
\left\|x_{k}-l, z_{1}, z_{2}, \ldots, z_{n-1}\right\|<\varepsilon, \quad \forall k \geq n_{0} \text { for every } z_{1}, z_{2}, \ldots, z_{n-1} \in X .
$$

Definition 2.2. A sequence $\left(x_{k}\right)$ in an $n$-normed space $(X,\|., \ldots,\|$.$) is said to be Cauchy with$ respect the $n$-norm if for each $\varepsilon>0$, there exists an positive integer $n_{0}=n_{0}(\varepsilon)$ such that

$$
\left\|x_{k}-x_{m}, z_{1}, z_{2}, \ldots, z_{n-1}\right\|<\varepsilon, \quad \forall k, m \geq n_{0} \text { for every } z_{1}, z_{2}, \ldots, z_{n-1} \in X \text {. }
$$

If every Cauchy sequence in $X$ converges to some $l \in X$, then $X$ is said to be complete with respect to the $n$-norm. Any complete $n$-normed space is said to be $n$-Banach space.

Definition 2.3. Let $S$ be a nonempty set. A nonempty family of sets $I \subseteq P(S)$ (power set of $S$ ) is called an ideal in $S$ if (i) for each $A, B \in I$, we have $A \cup B \in I$; (ii) for each $A \in I$ and $B \subseteq A$, we have $B \in I$. 
Definition 2.4. Let $S$ be a nonempty set. A family $F \subseteq P(S)$ (power set of $S$ ) is called a filter on $S$ if (i) $\emptyset \notin F$; (ii) for each $A, B \in F$, we have $A \cap B \in F$; (iii) for each $A \in F$ and $B \supseteq A$, we have $B \in F$.

An ideal $I$ is called nontrivial if $I \neq \emptyset$ and $S \notin I$. It is clear that $I \subseteq P(S)$ is a nontrivial ideal if and only if the class $F=F(I)=\{S-A: A \in I\}$ is a filter on $S$. The filter $F(I)$ is called the filter associated with the ideal $I$.

A nontrivial ideal $I \subseteq P(S)$ is called an admissible ideal in $S$ if and only if it contains all singletons, that is, if it contains $\{\{x\}: x \in S\}$.

Definition 2.5. A sequence $\left(x_{k}\right)$ in a normed space $(X,\|\cdot\|)$ is said to be statistically convergent to $x_{0} \in X$ if for each $\varepsilon>0$, the set $E(\varepsilon)=\left\{k \in \mathbb{N}:\left\|x_{k}-x_{0}\right\| \geq \varepsilon\right\}$ has natural density zero.

Definition 2.6. A sequence $\left(x_{k}\right)$ in a normed space $(X,\|\cdot\|)$ is said to be I-convergent to $x_{0} \in X$ if for each $\varepsilon>0$, the set

$$
E(\varepsilon)=\left\{k \in \mathbb{N}:\left\|x_{k}-x_{0}\right\| \geq \varepsilon\right\} \quad \text { belongs to } I .
$$

Definition 2.7. A sequence $\left(x_{k}\right)$ in a normed space $(X,\|\cdot\|)$ is said to be I-bounded if there exists $M>0$ such that the set $\left\{k \in \mathbb{N}:\left\|x_{k}\right\|>M\right\}$ belongs to $I$.

Definition 2.8. A sequence $\left(x_{k}\right)$ in a normed space $(X,\|\cdot\|)$ is said to be I-Cauchy if for each $\varepsilon>0$, there exists an positive integer $m=m(\varepsilon)$ such that the set

$$
E(\varepsilon)=\left\{k \in \mathbb{N}:\left\|x_{k}-x_{m}\right\| \geq \varepsilon\right\} \quad \text { belongs to } I \text {. }
$$

Definition 2.9. A sequence $\left(x_{k}\right)$ in an $n$-normed space $(X,\|\cdot, \ldots, \cdot\|)$ is said to be I-convergent to $x_{0} \in X$ with respect the $n$-norm, if for each $\varepsilon>0$, the set

$$
E(\varepsilon)=\left\{k \in \mathbb{N}:\left\|x_{k}-x_{0}, z_{1}, z_{2}, \ldots, z_{n-1}\right\| \geq \varepsilon, \text { for every } z_{1}, z_{2}, \ldots, z_{n-1} \in X\right\} \text { belongs to } I \text {. }
$$

Definition 2.10. A sequence $\left(x_{k}\right)$ in an $n$-normed space $(X,\|\cdot, \ldots, \cdot\|)$ is said to be $I$-Cauchy with respect to the $n$-norm if for each $\varepsilon>0$, there exists a positive integer $m=m(\varepsilon)$ such that the set

$$
E(\varepsilon)=\left\{k \in \mathbb{N}:\left\|x_{k}-x_{m}, z_{1}, z_{2}, \ldots, z_{n-1}\right\| \geq \varepsilon, \text { for every } z_{1}, z_{2}, \ldots, z_{n-1} \in X\right\} \text { belongs to } I \text {. }
$$

Definition 2.11. A sequence space $E$ is said to be normal (or solid) if $\left(\alpha_{k} x_{k}\right) \in E$, whenever $\left(x_{k}\right) \in E$ and for all sequence $\left(\alpha_{k}\right)$ of scalars with $\left|\alpha_{k}\right| \leq 1$, for all $k \in \mathbb{N}$.

Let $K=\left\{k_{1}<k_{2}<\cdots\right\} \subseteq \mathbb{N}$ and $E$ a sequence space. A $K$-step space of $E$ is a sequence space $\lambda_{K}^{E}=\left\{\left(x_{k_{n}}\right) \in w:\left(k_{n}\right) \in E\right\}$. 
A canonical preimage of a sequence $\left\{x_{k_{n}}\right\} \in \lambda_{K}^{E}$ is a sequence $\left\{y_{k}\right\} \in w$ defined as

$$
y_{k}= \begin{cases}x_{k}, & \text { if } k \in K \\ 0, & \text { otherwise }\end{cases}
$$

A canonical preimage of a step space $\lambda_{K}^{E}$ is a set of canonical preimages of all elements in $\lambda_{K}^{E}$; that is, $y$ is in canonical preimage of $\lambda_{K}^{E}$ if and only if $y$ is canonical preimage of some $x \in \lambda_{K}^{E}$.

Definition 2.12. A sequence space $E$ is said to be monotone if it contains the canonical preimages of all its step spaces.

Example 2.13. If we take $I=I_{f}=\{A \subseteq \mathbb{N}: A$ is a finite subset $\}$. Then, $I_{f}$ is a nontrivial admissible ideal of $\mathbb{N}$ and the corresponding convergence coincide with usual convergence of sequences in $n$-normed spaces.

Example 2.14. If we take $I=I_{\delta}=\{A \subseteq \mathbb{N}: \delta(A)=0\}$, where $\delta(A)$ denote the asymptotic density of the set $A$ then $I_{\delta}$ is a nontrivial admissible ideal of $\mathbb{N}$ and the corresponding convergence coincide with statistical convergence in $n$-normed spaces.

Now, we state the following lemmas (see [12] for details).

Lemma 2.15. Every n-normed space is an $(n-r)$-normed space for all $r=1,2, \ldots, n-1$. In particular, every n-normed space is a normed space.

Lemma 2.16. A standard n-normed space is complete if and only if it is complete with respect to the usual norm $\|\cdot\|=\sqrt{\langle,\rangle}$.

Lemma 2.17. On a standard $n$-normed space $X$, the derived $(n-1)$-norm $\|\cdot, \ldots, \cdot\|_{\infty}$ defined with respect to the orthogonal set $\left\{e_{1}, e_{2}, \ldots, e_{n}\right\}$ is equivalent to the standard $(n-1)$-norm $\|\cdot \ldots, \cdot\|_{S}$. To be precise, one has

$$
\left\|x_{1}, x_{2}, \ldots, x_{n-1}\right\|_{\infty} \leq\left\|x_{1}, x_{2}, \ldots, x_{n-1}\right\|_{S} \leq \sqrt{n}\left\|x_{1}, x_{2}, \ldots, x_{n-1}\right\|_{\infty}
$$

for all $x_{1}, x_{2}, \ldots, x_{n-1} \in X$, where $\left\|x_{1}, x_{2}, \ldots, x_{n-1}\right\|_{\infty}=\max _{1 \leq i \leq n}\left\{\left\|x_{1}, x_{2}, \ldots, x_{n-1}, e_{i}\right\|_{S}\right\}$.

Lemma 2.18 (Kamthan and Gupta [24]). Every normal space is monotone.

\section{Main Results}

In this section, we define some new ideal convergent sequence spaces and investigate their linear topological structures. Also, we find out some relations related to these sequence spaces. 
Let $I$ be an admissible ideal of $\mathbb{N}, M$ an Orlicz function, and $(X,\|\cdot, \ldots, \cdot\|)$ an $n$-normed space. Further, let $p=\left(p_{k}\right)$ be any bounded sequence of positive real numbers, and let $w(n-$ $X)$, denote the spaces of all $X$-valued sequences. For each $\varepsilon>0$, we define the following sequence spaces:

$$
\begin{aligned}
& W^{I}\left(\Delta_{m}^{s}, M, p,\|\cdot, \ldots,\|\right) \\
& =\left\{\left(x_{k}\right) \in w(n-X):\left\{n \in \mathbb{N}: \frac{1}{n} \sum_{k=1}^{n}\left[M\left(\left\|\frac{\Delta_{m}^{s} x_{k}-1}{\rho}, z_{1} z_{2}, \ldots, z_{n-1}\right\|\right)\right]^{p_{k}} \geq \varepsilon\right\} \in I,\right. \\
& \left.\quad \text { for some } \rho>0, l \in X \text { and } \forall z_{1}, z_{2} \ldots z_{n-1} \in X\right\},
\end{aligned}
$$$$
W^{I}\left(\Delta_{m}^{s}, M, p,\|\cdot, \ldots, \cdot\|\right)
$$$$
=\left\{\left(x_{k}\right) \in w(n-X):\left\{n \in \mathbb{N}: \frac{1}{n} \sum_{k=1}^{n}\left[M\left(\left\|\frac{\Delta_{m}^{s} x_{k}}{\rho}, z_{1} z_{2}, \ldots, z_{n-1}\right\|\right)\right]^{p_{k}} \geq \varepsilon\right\} \in I,\right.
$$

for some $\rho>0$ and $\left.\forall z_{1}, z_{2} \ldots z_{n-1} \in X\right\}$,

$$
\begin{aligned}
& W^{I}\left(\Delta_{m}^{s}, M, p,\|\cdot, \ldots, \cdot\|\right) \\
& =\left\{\left(x_{k}\right) \in w(n-X): \sup _{n \in \mathbb{N}} \frac{1}{n} \sum_{k=1}^{n}\left[M\left(\left\|\frac{\Delta_{m}^{s} x_{k}}{\rho}, z_{1} z_{2}, \ldots, z_{n-1}\right\|\right)\right]^{p_{k}}<\infty,\right.
\end{aligned}
$$

$$
\begin{aligned}
& \left.\quad \text { for some } \rho>0 \text { and } \forall z_{1}, z_{2} \ldots z_{n-1} \in X\right\}, \\
& W^{I}\left(\Delta_{m}^{s}, M, p,\|\cdot \ldots, \cdot\|\right) \\
& =\left\{\left(x_{k}\right) \in w(n-X): \exists K>0 \text { s.t. }\left\{n \in \mathbb{N}: \frac{1}{n} \sum_{k=1}^{n}\left[M\left(\left\|\frac{\Delta_{m}^{s} x_{k}}{\rho}, z_{1} z_{2}, \ldots, z_{n-1}\right\|\right)\right]^{p_{k}} \geq K\right\} \in I,\right. \\
& \left.\quad \text { for some } \rho>0 \text { and } \forall z_{1}, z_{2} \ldots z_{n-1} \in X\right\} .
\end{aligned}
$$

The following well-known inequality will be used throughout the article. Let $p=\left(p_{k}\right)$ be any sequence of positive real numbers with $0 \leq p_{k} \leq \sup _{k} p_{k}=G, D=\max \left\{1,2^{G-1}\right\}$, then $\left|a_{k}+b_{k}\right|^{p_{k}} \leq D\left(\left|a_{k}\right|^{p_{k}}+\left|b_{k}\right|^{p_{k}}\right)$, for all $k \in \mathbb{N}$ and $a_{k}, b_{k} \in \mathbb{C}$. Also, $|a|^{p_{k}} \leq \max \left\{1,|a|^{G}\right\}$, for all $a \in \mathbb{C}$.

Theorem 3.1. The spaces $W^{I}\left(\Delta_{m}^{s}, M, p,\|\cdot, \ldots, \cdot\|\right), W_{0}^{I}\left(\Delta_{m}^{s}, M, p,\|\cdot, \ldots, \cdot\|\right)$ and $W_{\infty}^{I}\left(\Delta_{m}^{s}, M, p\right.$, $\|\cdot, \ldots, \cdot\|)$ are linear.

Proof. We will prove the result for the space $W_{0}^{I}\left(\Delta_{m}^{s}, M, p,\|\cdot, \ldots, \cdot\|\right)$ only and the others can be proved similarly. 
Let $x=\left(x_{k}\right)$ and $y=\left(y_{k}\right)$ be any two elements of the space $W_{0}^{I}\left(\Delta_{m}^{s}, M, p,\|\cdot, \ldots, \cdot\|\right)$. Then, there exist $\rho_{1}>0$ and $\rho_{2}>0$ such that the sets

$$
\begin{aligned}
& A_{\varepsilon / 2}(x)=\left\{n \in \mathbb{N}: \frac{1}{n} \sum_{k=1}^{n}\left[M\left(\left\|\frac{\Delta_{m}^{s} x_{k}}{\rho_{1}}, z_{1}, z_{2}, \ldots, z_{n-1}\right\|\right)\right]^{p_{k}} \geq \frac{\varepsilon}{2},\right. \\
&\text { for every } \left.z_{1}, z_{2}, \ldots, z_{n-1} \in X\right\}, \\
& B_{\varepsilon / 2}(y)=\left\{n \in \mathbb{N}: \frac{1}{n} \sum_{k=1}^{n}\left[M\left(\left\|\frac{\Delta_{m}^{s} y_{k}}{\rho_{2}}, z_{1}, z_{2}, \ldots, z_{n-1}\right\|\right)\right]^{p_{k}} \geq \frac{\varepsilon}{2},\right. \\
&\text { for every } \left.z_{1}, z_{2}, \ldots, z_{n-1} \in X\right\}
\end{aligned}
$$

belong to $I$.

Let $\alpha, \beta \in \mathbb{R}$ be two scalars. Since $\|\cdot, \ldots, \cdot\|$ is an $n$-norm, the operator $\Delta^{s}$ is linear and the Orlicz function $M$ is continuous, the following inequality holds:

$$
\begin{aligned}
\frac{1}{n} \sum_{k=1}^{n} & {\left[M\left(\left\|\frac{\Delta_{m}^{s}\left(\alpha x_{k}+\beta y_{k}\right)}{\left(|\alpha| \rho_{1}+|\beta| \rho_{2}\right)}, z_{1}, z_{2}, \ldots, z_{n-1}\right\|\right)\right]^{p_{k}} } \\
\leq & D \frac{1}{n} \sum_{k=1}^{n}\left[\frac{|\alpha|}{\left(|\alpha| \rho_{1}+|\beta| \rho_{2}\right)} M\left(\left\|\frac{\Delta_{m}^{s} x_{k}}{\rho_{1}}, z_{1}, z_{2}, \ldots, z_{n-1}\right\|\right)\right]^{p_{k}} \\
& +D \frac{1}{n} \sum_{k=1}^{n}\left[\frac{|\beta|}{\left(|\alpha| \rho_{1}+|\beta| \rho_{2}\right)} M\left(\left\|\frac{\Delta_{m}^{s} y_{k}}{\rho_{2}}, z_{1}, z_{2}, \ldots, z_{n-1}\right\|\right)\right]^{p_{k}} \\
\leq & D K \frac{1}{n} \sum_{k=1}^{n}\left[M\left(\left\|\frac{\Delta_{m}^{s} x_{k}}{\rho_{1}}, z_{1}, z_{2}, \ldots, z_{n-1}\right\|\right)\right]^{p_{k}} \\
& +D K \frac{1}{n} \sum_{k=1}^{n}\left[M\left(\left\|\frac{\Delta_{m}^{s} y_{k}}{\rho_{2}}, z_{1}, z_{2}, \ldots, z_{n-1}\right\|\right)\right]^{p_{k}},
\end{aligned}
$$

where $K=\max \left\{1,\left(|\alpha| /\left(|\alpha| \rho_{1}+|\beta| \rho_{2}\right)\right),\left(|\beta| /\left(|\alpha| \rho_{1}+|\beta| \rho_{2}\right)\right)\right\}$.

From the above relation, we get

$$
\begin{aligned}
\{n & \left.\in \mathbb{N}: \frac{1}{n} \sum_{k=1}^{n}\left[M\left(\left\|\frac{\Delta_{m}^{s}\left(\alpha x_{k}+\beta y_{k}\right)}{\left(|\alpha| \rho_{1}+|\beta| \rho_{2}\right)}, z_{1}, z_{2}, \ldots, z_{n-1}\right\|\right)\right]^{p_{k}} \geq \varepsilon\right\} \\
& \subseteq\left\{n \in \mathbb{N}: D K \frac{1}{n} \sum_{k=1}^{n}\left[M\left(\left\|\frac{\Delta_{m}^{s} x_{k}}{\rho_{1}}, z_{1}, z_{2}, \ldots, z_{n-1}\right\|\right)\right]^{p_{k}} \geq \frac{\varepsilon}{2}\right\} \\
& \cup\left\{n \in \mathbb{N}: D K \frac{1}{n} \sum_{k=1}^{n}\left[M\left(\left\|\frac{\Delta_{m}^{s} y_{k}}{\rho_{2}}, z_{1}, z_{2}, \ldots, z_{n-1}\right\|\right)\right]^{p_{k}} \geq \frac{\varepsilon}{2}\right\} .
\end{aligned}
$$


Since both the sets on the right hand side of the relation (3.7) belong to $I$, this completes the proof.

Note 1. It is easy to verify that the space $W_{\infty}\left(\Delta_{m}^{s}, M, p,\|\cdot, \ldots, \cdot\|\right)$ is a linear space.

Theorem 3.2. The space $W^{I}\left(\Delta_{m}^{s}, M, p,\|\cdot, \ldots, \cdot\|\right), \quad W_{0}^{I}\left(\Delta_{m}^{s}, M, p,\|\cdot, \ldots, \cdot\|\right), \quad W_{\infty}^{I}\left(\Delta_{m}^{s}, M, p\right.$, $\|\cdot, \ldots, \cdot\|)$, and $W_{\infty}\left(\Delta_{m}^{s}, M, p,\|\cdot, \ldots, \cdot\|\right)$ are paranormed spaces (not totally paranormed) with respect to the paranorm $g_{\Delta}$ defined by

$$
\begin{aligned}
& g_{\Delta}(x)=\sum_{k=1}^{m s}\left\|x_{k}, z_{1}, z_{2}, \ldots, z_{n-1}\right\| \\
& +\inf \left\{\rho^{p_{k} / H}: \sup _{k} M\left(\left\|\frac{\Delta_{m}^{s} x_{k}}{\rho}, z_{1}, z_{2}, \ldots, z_{n-1}\right\|\right) \leq 1,\right. \\
& \text { for some } \left.\rho>0 \text { and } \forall z_{1}, z_{2}, \ldots, z_{n-1} \in X\right\}
\end{aligned}
$$

where $H=\max \left\{1\right.$, sup $\left.p_{k}\right\}$.

Proof. Clearly, $g_{\Delta}(-x)=g_{\Delta}(x)$ and $g_{\Delta}(\theta)=0$. Let $x=\left(x_{k}\right)$ and $y=\left(y_{k}\right)$ be any two elements of the space $Z\left(\Delta_{m}^{s}, M, p,\|\cdot, \ldots, \cdot\|\right)$, where $Z=W^{I}, W_{0}^{I}, W_{\infty}^{I}$ and $W_{\infty}$.

Then,for $\rho>0$, we set

$$
\begin{aligned}
& A_{1}=\left\{\rho>0: \sup _{k} M\left(\left\|\frac{\Delta_{m}^{s} x_{k}}{\rho}, z_{1}, z_{2}, \ldots, z_{n-1}\right\|\right) \leq 1, \forall z_{1}, z_{2}, \ldots, z_{n-1} \in X\right\}, \\
& A_{2}=\left\{\rho>0: \sup _{k} M\left(\left\|\frac{\Delta_{m}^{s} y_{k}}{\rho}, z_{1}, z_{2}, \ldots, z_{n-1}\right\|\right) \leq 1, \forall z_{1}, z_{2}, \ldots, z_{n-1} \in X\right\} .
\end{aligned}
$$

Let $\rho_{1} \in A_{1}$ and $\rho_{2} \in A_{2}$. If $\rho=\rho_{1}+\rho_{2}$, then we have

$$
\begin{aligned}
& M\left(\left\|\frac{\Delta_{m}^{s}\left(x_{k}+y_{k}\right)}{\rho}, z_{1}, z_{2}, \ldots, z_{n-1}\right\|\right) \\
& \quad \leq \frac{\rho_{1}}{\rho_{1}+\rho_{2}} M\left(\left\|\frac{\Delta_{m}^{s} x_{k}}{\rho_{1}}, z_{1}, z_{2}, \ldots, z_{n-1}\right\|\right)+\frac{\rho_{2}}{\rho_{1}+\rho_{2}} M\left(\left\|\frac{\Delta_{m}^{s} y_{k}}{\rho_{2}}, z_{1}, z_{2}, \ldots, z_{n-1}\right\|\right) .
\end{aligned}
$$


Thus, we have

$$
\begin{aligned}
& \sup _{k}\left[M\left(\left\|\frac{\Delta_{m}^{s}\left(x_{k}+y_{k}\right)}{\rho}, z_{1}, z_{2}, \ldots, z_{n-1}\right\|\right)\right]^{p_{k}} \leq 1, \\
g_{\Delta}(x+y)= & \sum_{k=1}^{m s}\left\|x_{k}+y_{k}, z_{1}, z_{2}, \ldots, z_{n-1}\right\|+\inf \left\{\left(\rho_{1}+\rho_{2}\right)^{p_{k} / H}: \rho_{1} \in A_{1}, \rho_{2} \in A_{2}\right\} \\
\leq & \sum_{k=1}^{m s}\left\|x_{k}, z_{1}, z_{2}, \ldots, z_{n-1}\right\|+\inf \left\{\left(\rho_{1}\right)^{p_{k} / H}: \rho_{1} \in A_{1}\right\} \\
& +\sum_{k=1}^{m s}\left\|y_{k}, z_{1}, z_{2}, \ldots, z_{n-1}\right\| \inf \left\{\left(\rho_{2}\right)^{p_{k} / H}: \rho_{2} \in A_{2}\right\}=g_{\Delta}(x)+g_{\Delta}(y) .
\end{aligned}
$$

Let $\lambda^{s} \rightarrow \lambda$, where $\lambda^{s}, \lambda \in \mathbb{C}$, and let $g_{\Delta}\left(x^{s}-x\right) \rightarrow 0$ as $s \rightarrow \infty$. We have to show that $g_{\Delta}\left(\lambda^{s} x^{s}-\lambda x\right) \rightarrow 0$ as $s \rightarrow \infty$.

We set

$$
\begin{gathered}
A_{3}=\left\{\rho_{s}>0: \sup _{k}\left[M\left(\left\|\frac{\Delta_{m}^{s} x_{k}^{s}}{\rho_{s}}, z_{1}, z_{2}, \ldots, z_{n-1}\right\|\right)\right]^{p_{k}} \leq 1, \forall z_{1}, z_{2}, \ldots, z_{n-1} \in X\right\}, \\
A_{4}=\left\{\rho_{s}^{\prime}>0: \sup _{k}\left[M\left(\left\|\frac{\Delta_{m}^{s}\left(x_{k}^{s}-x_{k}\right)}{\rho_{s}^{\prime}}, z_{1}, z_{2}, \ldots, z_{n-1}\right\|\right)\right]^{p_{k}} \leq 1, \forall z_{1}, z_{2}, \ldots, z_{n-1} \in X\right\} .
\end{gathered}
$$

If $\rho_{s} \in A_{3}$ and $\rho_{s}{ }^{\prime} \in A_{4}$, we observe by the continuity of the Orlicz function $M$ that

$$
\begin{aligned}
M( & \left.\left\|\frac{\Delta_{m}^{s}\left(\lambda^{s} x_{k}^{s}-\lambda x_{k}\right)}{\left(\left|\lambda^{s}-\lambda\right| \rho_{s}+|\lambda| \rho_{s}^{\prime}\right)}, z_{1}, z_{2}, \ldots, z_{n-1}\right\|\right) \\
\leq & M\left(\left\|\frac{\Delta_{m}^{s}\left(\lambda^{s} x_{k}^{s}-\lambda x_{k}^{s}\right)}{\left(\left|\lambda^{s}-\lambda\right| \rho_{s}+|\lambda| \rho_{s}^{\prime}\right)}, z_{1}, z_{2}, \ldots, z_{n-1}\right\|\right) \\
& +M\left(\left\|\frac{\Delta_{m}^{s}\left(\lambda x_{k}^{s}-\lambda x_{k}\right)}{\left(\left|\lambda^{s}-\lambda\right| \rho_{s}+|\lambda| \rho_{s}^{\prime}\right)}, z_{1}, z_{2}, \ldots, z_{n-1}\right\|\right) \\
\leq & \frac{\left|\lambda^{s}-\lambda\right| \rho_{s}}{\left|\lambda^{s}-\lambda\right| \rho_{s}+|\lambda| \rho_{s}^{\prime}} M\left(\left\|\frac{\Delta_{m}^{s}\left(x_{k}^{s}\right)}{\rho_{s}}, z_{1}, z_{2}, \ldots, z_{n-1}\right\|\right) \\
& +\frac{|\lambda| \rho_{s}^{\prime}}{\left|\lambda^{s}-\lambda\right| \rho_{s}+|\lambda| \rho_{s}^{\prime}} M\left(\left\|\frac{\Delta_{m}^{s}\left(x_{k}^{s}-x_{k}\right)}{\rho_{s}^{\prime}}, z_{1}, z_{2}, \ldots, z_{n-1}\right\|\right) .
\end{aligned}
$$

From the above inequality, it follows that

$$
\sup _{k}\left[M\left(\left\|\frac{\Delta_{m}^{s}\left(\lambda^{s} x_{k}^{s}-\lambda x_{k}\right)}{\left(\left|\lambda^{s}-\lambda\right| \rho_{s}+|\lambda| \rho_{s}^{\prime}\right)}, z_{1}, z_{2}, \ldots, z_{n-1}\right\|\right)\right]^{p_{k}} \leq 1
$$


and consequently

$$
\begin{aligned}
& g_{\Delta}\left(\lambda^{s} x^{s}-\lambda x\right) \\
& =\sum_{k=1}^{m s}\left\|\lambda^{s} x_{k}^{s}-\lambda x_{k}, z_{1}, z_{2}, \ldots, z_{n-1}\right\|+\inf \left\{\left(\left|\lambda^{s}-\lambda\right| \rho_{s}+|\lambda| \rho_{s}^{\prime}\right)^{p_{k} / H}: \rho_{s} \in A_{3}, \rho_{s}^{\prime} \in A_{4}\right\} \\
& \leq\left|\lambda^{s}-\lambda\right| \sum_{k=1}^{m s}\left\|x_{k^{s}}^{s} z_{1}, z_{2}, \ldots, z_{n-1}\right\|+\left|\lambda^{s}-\lambda\right|^{p_{k} / H} \inf \left\{\left(\rho_{s}\right)^{p_{k} / H}: \rho_{s} \in A_{3}\right\} \\
& \quad+|\lambda| \sum_{k=1}^{m s}\left\|x_{k}^{s}-x_{k}, z_{1}, z_{2}, \ldots, z_{n-1}\right\|+|\lambda|^{p_{k} / H} \inf \left\{\left(\rho_{s}^{\prime}\right)^{p_{k} / H}: \rho_{s}^{\prime} \in A_{4}\right\} \\
& \leq \max \left\{\left|\lambda^{s}-\lambda\right|,\left|\lambda^{s}-\lambda\right|^{p_{k} / H}\right\} g_{\Delta}\left(x^{s}\right)+\max \left\{|\lambda|,|\lambda|^{p_{k} / H}\right\} g_{\Delta}\left(x^{s}-x\right) .
\end{aligned}
$$

Note that $g_{\Delta}\left(x^{s}\right) \leq g_{\Delta}(x)+g_{\Delta}\left(x^{s}-x\right)$, for all $s \in \mathbb{N}$.

Hence, by our assumption, the right hand side (3.17) tends to 0 as $s \rightarrow \infty$, and the result follows. This completes the proof of the theorem.

Theorem 3.3. Let $M, M_{1}$, and $M_{2}$ be Orlicz functions. Then, the following hold:

(a) $W_{0}^{I}\left(\Delta_{m}^{s}, M, p,\|\cdot, \ldots, \cdot\|\right) \subseteq W_{0}^{I}\left(\Delta_{m}^{s}, M . M_{1}, p,\|\cdot, \ldots, \cdot\|\right)$, provided $\left(p_{k}\right)$ be such that $G_{0}=$ inf $p_{k}>0$,

(b) $W_{0}^{I}\left(\Delta_{m}^{s}, M_{1}, p,\|\cdot \ldots, \cdot\|\right) \cap W_{0}^{I}\left(\Delta_{m}^{s}, M_{2}, p,\|\cdot, \ldots, \cdot\|\right) \subseteq W_{0}^{I}\left(\Delta_{m}^{s}, M_{1}+M_{2}, p,\|\cdot, \ldots, \cdot\|\right)$.

Proof. (a) Let $\varepsilon>0$ be given. Choose $\varepsilon_{1}>0$ such that $\max \left\{\varepsilon_{1}^{G}, \varepsilon_{1}^{G_{0}}\right\}<\varepsilon$.

Using the continuity of the Orlicz function $M$, choose $0<\delta<1$ such that $0<t<\delta$ implies that $M(t)<\varepsilon_{1}$.

Let $x=\left(x_{k}\right)$ be any element in $W_{0}^{I}\left(\Delta_{m}^{s}, M_{1}, p,\|\cdot, \ldots, \cdot\|\right)$. Put

$$
A_{\delta}=\left\{n \in \mathbb{N}: \frac{1}{n} \sum_{k=1}^{n}\left[M_{1}\left(\left\|\frac{\Delta_{m}^{s} x_{k}}{\rho}, z_{1}, z_{2}, \ldots, z_{n-1}\right\|\right)\right]^{p_{k}} \geq \delta^{G}\right\} .
$$

Then, by definition of ideal, we have the set $A_{\delta} \in I$.

If $n \notin A_{\delta}$, then we have

$$
\begin{aligned}
\frac{1}{n} \sum_{k=1}^{n}\left[M_{1}\left(\left\|\frac{\Delta_{m}^{s} x_{k}}{\rho}, z_{1}, z_{2}, \ldots, z_{n-1}\right\|\right)\right]^{p_{k}}<\delta^{G} \\
\Longrightarrow \sum_{k=1}^{n}\left[M_{1}\left(\left\|\frac{\Delta_{m}^{s} x_{k}}{\rho}, z_{1}, z_{2}, \ldots, z_{n-1}\right\|\right)\right]^{p_{k}}<n \delta^{G} \\
\Longrightarrow\left[M_{1}\left(\left\|\frac{\Delta_{m}^{s} x_{k}}{\rho}, z_{1}, z_{2}, \ldots, z_{n-1}\right\|\right)\right]^{p_{k}}<\delta^{G}, \quad \forall k=1,2, \ldots, n \\
\Longrightarrow M_{1}\left(\left\|\frac{\Delta_{m}^{s} x_{k}}{\rho}, z_{1}, z_{2}, \ldots, z_{n-1}\right\|\right)<\delta, \quad \forall k=1,2, \ldots, n .
\end{aligned}
$$


Using the continuity of the Orlicz function $M$, then from the relation (3.19), we have

$$
\sum_{k=1}^{n} M\left(M_{1}\left(\left\|\frac{\Delta_{m}^{s} x_{k}}{\rho}, z_{1}, z_{2}, \ldots, z_{n-1}\right\|\right)\right)<\varepsilon_{1}, \quad \forall k=1,2, \ldots, n
$$

Consequently, we get

$$
\begin{gathered}
\sum_{k=1}^{n}\left[M\left(M_{1}\left(\left\|\frac{\Delta_{m}^{s} x_{k}}{\rho}, z_{1}, z_{2}, \ldots, z_{n-1}\right\|\right)\right)\right]^{p_{k}}<n \max \left\{\varepsilon_{1}^{G}, \varepsilon_{1}^{G_{0}}\right\}<n \varepsilon \\
\Longrightarrow \frac{1}{n} \sum_{k=1}^{n}\left[M\left(M_{1}\left(\left\|\frac{\Delta_{m}^{s} x_{k}}{\rho}, z_{1}, z_{2}, \ldots, z_{n-1}\right\|\right)\right)\right]^{p_{k}}<\varepsilon .
\end{gathered}
$$

This shows that

$$
\left\{n \in \mathbb{N}: \frac{1}{n} \sum_{k=1}^{n}\left[M\left(M_{1}\left(\left\|\frac{\Delta_{m}^{s} x_{k}}{\rho}, z_{1}, z_{2}, \ldots, z_{n-1}\right\|\right)\right)\right]^{p_{k}} \geq \varepsilon,\right\} \subseteq A_{\delta} \in I
$$

This proves the result.

(b) Let $x=\left(x_{k}\right) \in W_{0}^{I}\left(\Delta_{m}^{s}, M_{1}, p,\|\cdot, \ldots, \cdot\|\right) \cap W_{0}^{I}\left(\Delta_{m}^{s}, M_{2}, p,\|\cdot, \ldots, \cdot\|\right)$. Then, by the following inequality, the result follows:

$$
\begin{aligned}
\frac{1}{n} \sum_{k=1}^{n}[( & \left.\left.M_{1}+M_{2}\right)\left(\left\|\frac{\Delta_{m}^{s} x_{k}}{\rho}, z_{1}, z_{2}, \ldots, z_{n-1}\right\|\right)\right]^{p_{k}} \\
\leq & D \frac{1}{n} \sum_{k=1}^{n}\left[M_{1}\left(\left\|\frac{\Delta_{m}^{s} x_{k}}{\rho}, z_{1}, z_{2}, \ldots, z_{n-1}\right\|\right)\right]^{p_{k}} \\
& +D \frac{1}{n} \sum_{k=1}^{n}\left[M_{2}\left(\left\|\frac{\Delta_{m}^{s} x_{k}}{\rho}, z_{1}, z_{2}, \ldots, z_{n-1}\right\|\right)\right]^{p_{k}} .
\end{aligned}
$$

Theorem 3.4. The inclusions $Z\left(\Delta_{m}^{s-1}, M, p,\|\cdot, \ldots, \cdot\|\right) \subseteq Z\left(\Delta_{m}^{s}, M, p,\|\cdot, \ldots, \cdot\|\right)$ are strict for $s \geq$ 1. In general, $Z\left(\Delta_{m}^{j}, M, p,\|\cdot, \ldots, \cdot\|\right) \subseteq Z\left(\Delta_{m}^{s}, M, p,\|\cdot \ldots, \cdot\|\right)$, for $j=0,1,2, \ldots, s-1$, and the inclusion is strict, where $Z=W^{I}, W_{0}^{I}$, and $W_{\infty}^{I}$.

Proof. We will give the proof for $W_{0}^{I}\left(\Delta_{m}^{s}, M, p,\|\cdot, \ldots, \cdot\|\right)$ only. The others can be proved by similar arguments.

Let $x=\left(x_{k}\right)$ be any element in the space $W_{0}^{I}\left(\Delta_{m}^{s}, M, p,\|\cdot, \ldots, \cdot\|\right)$. Let $\varepsilon>0$ be given. Then, there exists $\rho>0$ such that the set

$$
\left\{n \in \mathbb{N}: \frac{1}{n} \sum_{k=1}^{n}\left[M\left(\left\|\frac{\Delta_{m}^{s} x_{k}}{\rho}, z_{1} z_{2}, \ldots, z_{n-1}\right\|\right)\right]^{p_{k}} \geq \varepsilon\right\} \in I
$$


Since $M$ is nondecreasing and convex, it follows that

$$
\begin{aligned}
& \frac{1}{n} \sum_{k=1}^{n}\left.M\left(\left\|\frac{\Delta_{m}^{s} x_{k}}{2 \rho}, z_{1} z_{2}, \ldots, z_{n-1}\right\|\right)\right]^{p_{k}} \\
&= \frac{1}{n} \sum_{k=1}^{n}\left[M\left(\left\|\frac{\Delta_{m}^{s-1} x_{k+1}-\Delta_{m}^{s-1} x_{k}}{2 \rho}, z_{1} z_{2}, \ldots, z_{n-1}\right\|\right)\right]^{p_{k}} \\
& \leq \frac{D}{n} \sum_{k=1}^{n}\left[\frac{1}{2} M\left(\left\|\frac{\Delta_{m}^{s-1} x_{k+1}}{\rho}, z_{1} z_{2}, \ldots, z_{n-1}\right\|\right)\right]^{p_{k}} \\
& \quad+\frac{D}{n} \sum_{k=1}^{n}\left[\frac{1}{2} M\left(\left\|\frac{\Delta_{m}^{s-1} x_{k}}{\rho}, z_{1} z_{2}, \ldots, z_{n-1}\right\|\right)\right]^{p_{k}} \\
& \leq \frac{D H}{n} \sum_{k=1}^{n}\left(\left[M\left(\left\|\frac{\Delta_{m}^{s-1} x_{k+1}}{\rho}, z_{1} z_{2}, \ldots, z_{n-1}\right\|\right)\right]^{p_{k}}+\left[M\left(\left\|\frac{\Delta_{m}^{s-1} x_{k}}{\rho}, z_{1} z_{2}, \ldots, z_{n-1}\right\|\right)\right]^{p_{k}}\right),
\end{aligned}
$$

where $H=\max \left\{1,(1 / 2)^{G}\right\}$.

Thus, we have

$$
\begin{aligned}
\{n \in \mathbb{N}: & \left.\frac{1}{n} \sum_{k=1}^{n}\left[M\left(\left\|\frac{\Delta_{m}^{s} x_{k}}{2 \rho}, z_{1} z_{2}, \ldots, z_{n-1}\right\|\right)\right]^{p_{k}} \geq \varepsilon\right\} \\
& \subseteq\left\{n \in \mathbb{N}: \frac{D H}{n} \sum_{k=1}^{n}\left[M\left(\left\|\frac{\Delta_{m}^{s-1} x_{k+1}}{\rho}, z_{1} z_{2}, \ldots, z_{n-1}\right\|\right)\right]^{p_{k}} \geq \frac{\varepsilon}{2}\right\} \\
& \cup\left\{n \in \mathbb{N}: \frac{D H}{n} \sum_{k=1}^{n}\left[M\left(\left\|\frac{\Delta_{m}^{s-1} x_{k}}{\rho}, z_{1} z_{2}, \ldots, z_{n-1}\right\|\right)\right]^{p_{k}} \geq \frac{\varepsilon}{2}\right\} .
\end{aligned}
$$

Since both the sets in the right side of the relation (3.26) belongs to $I$, we get the set

$$
\left\{n \in \mathbb{N}: \frac{1}{n} \sum_{k=1}^{n}\left[M\left(\left\|\frac{\Delta_{m}^{s} x_{k}}{2 \rho}, z_{1} z_{2}, \ldots, z_{n-1}\right\|\right)\right]^{p_{k}} \geq \varepsilon\right\} \in I
$$

Let $M(x)=x^{2}$, for all $x \in[0, \infty), p_{k}=1$, for all $k \in \mathbb{N}$. Consider a sequence $x=\left(x_{k}\right)=$ $\left((k+1)^{r}\right)$. Then, $x$ belongs to $W_{0}^{I}\left(\Delta_{m}^{s-1}, M, p,\|\cdot, \ldots, \cdot\|\right)$ but does not belong to $W_{0}^{I}\left(\Delta_{m}^{s}, M, p\right.$, $\|\cdot, \ldots, \cdot\|)$. This shows that the inclusion $W_{0}^{I}\left(\Delta_{m}^{s-1}, M, p,\|\cdot, \ldots, \cdot\|\right) \subset W_{0}^{I}\left(\Delta_{m}^{s}, M, p,\|\cdot, \ldots, \cdot\|\right)$ is strict.

Theorem 3.5. Let $0<p_{k} \leq q_{k}$ and $\left(q_{k} / p_{k}\right)$ be bounded, then

$$
W^{I}\left(\Delta_{m}^{s}, M, q,\|\cdot, \ldots, \cdot\|\right) \subseteq W^{I}\left(\Delta_{m}^{s}, M, p,\|\cdot, \ldots, \cdot\|\right) .
$$


Proof. Let $x=\left(x_{k}\right) \in W^{I}\left(\Delta_{m}^{s}, M, q,\|\cdot, \ldots, \cdot\|\right)$. We put

$$
\begin{array}{r}
\left.y_{k}=\left(M\left(\left\|\frac{\Delta_{m}^{s} x_{k}-l}{\rho}, z_{1}, z_{2}, \ldots, z_{n-1}\right\|\right)\right)\right)^{q_{k}} \text { and } \beta_{k}=\frac{p_{k}}{q_{k}} \\
\forall k \in \mathbb{N} . \quad \text { Then, } 0<\beta_{k} \leq 1 .
\end{array}
$$

For all $k \in \mathbb{N}$. Let $\beta$ be such that $0<\beta<\beta_{k}$, for all $k \in \mathbb{N}$.

Define the sequences $\left(a_{k}\right)$ and $\left(b_{k}\right)$ as follows: for $y_{k} \geq 1$, let $a_{k}=y_{k}$ and $b_{k}=0$; for $y_{k}<1$, let $a_{k}=0$ and $b_{k}=y_{k}$.

Then, clearly, for all $k \in \mathbb{N}$, we have $y_{k}=a_{k}+b_{k}, y_{k}^{\beta_{k}}=a_{k}^{\beta_{k}}+b_{k}^{\beta_{k}} ; a_{k}^{\beta_{k}} \leq a_{k} \leq y_{k}$ and $b_{k}^{\beta_{k}} \leq b_{k}^{\beta}$. Therefore, we have

$$
\frac{1}{n} \sum_{k=1}^{n} y_{k}^{\beta_{k}} \leq \frac{1}{n} \sum_{k=1}^{n} y_{k} \leq\left[\frac{1}{n} \sum_{k=1}^{n} b_{k}\right]^{\beta}
$$

Hence, $\left(x_{k}\right) \in W^{I}\left(\Delta_{m}^{s}, M, p,\|\cdot, \ldots, \cdot\|\right)$.

Theorem 3.6. For any two sequences $p=\left(p_{k}\right)$ and $q=\left(q_{k}\right)$ of positive real numbers and for any two $n$-norms $\|\cdot, \ldots, \cdot\|_{1}$ and $\|\cdot, \ldots, \cdot\|_{2}$ on $X$, the following holds:

$$
Z\left(\Delta_{m}^{s}, M, p,\|\cdot, \ldots, \cdot\|_{1}\right) \cap Z\left(\Delta_{m}^{s}, M, p,\|\cdot, \ldots, \cdot\|_{2}\right) \neq \emptyset,
$$

where $Z=W^{I}, W_{0}^{I}, W_{\infty}^{I}$, and $W_{\infty}$.

Proof. Proof of the theorem is obvious, because the zero element belongs to each of the sequence spaces involved in the intersection.

Theorem 3.7. The sequence spaces $Z\left(\Delta_{m}^{s}, M, p,\|\cdot, \ldots, \cdot\|\right)$ are normal as well as monotone, where $Z=W_{0}^{I}, W_{\infty}^{I}$.

Proof. We will give the proof for $W_{0}^{I}\left(\Delta_{m}^{s}, M, p,\|\cdot, \ldots, \cdot\|\right)$ only. Let $x=\left(x_{k}\right)$ be any element in $W_{0}^{I}\left(\Delta_{m}^{s}, M, p,\|\cdot, \ldots, \cdot\|\right)$, and let $\left(\alpha_{k}\right)$ be a sequence of scalars such that $\left|\alpha_{k}\right| \leq 1$, for all $k \in \mathbb{N}$. Then, we have

$$
\begin{aligned}
& \left\{n \in \mathbb{N}: \frac{1}{n} \sum_{k=1}^{n}\left[M\left(\left\|\frac{\Delta^{s}\left(\alpha_{k} x_{k}\right)}{\rho}, z_{1} z_{2}, \ldots, z_{n-1}\right\|\right)\right]^{p_{k}} \geq \varepsilon\right\} \\
& \subseteq\left\{n \in \mathbb{N}: \frac{E}{n} \sum_{k=1}^{n}\left[M\left(\left\|\frac{\Delta^{s} x_{k}}{\rho}, z_{1} z_{2}, \ldots, z_{n-1}\right\|\right)\right]^{p_{k}} \geq \varepsilon\right\},
\end{aligned}
$$

where $E=\max \left\{1,\left|\alpha_{k}\right|^{G}\right\}$.

Hence, $\left(\alpha_{k} x_{k}\right) \in W_{0}^{I}\left(\Delta_{m}^{s}, M, p,\|\cdot, \ldots, \cdot\|\right)$, for all sequence $\left(\alpha_{k}\right)$ of scalars with $\left|\alpha_{k}\right| \leq 1$, for all $k \in \mathbb{N}$, whenever $\left(x_{k}\right) \in W_{0}^{I}\left(\Delta_{m}^{s}, M, p,\|\cdot \ldots, \cdot\|\right)$.

By Lemma 2.18, we have the space $W_{0}^{I}\left(\Delta_{m}^{S}, M, p,\|\cdot, \ldots, \cdot\|\right)$ is monotone. 
Remark 3.8. If we replace the difference operator $\Delta_{m}^{s}$ by $\Delta_{m}^{(s)}$, then for each $\varepsilon>0$, we get the following sequence spaces:

$$
\begin{aligned}
& W^{I}\left(\Delta_{m}^{(s)}, M, p,\|\cdot, \ldots, \cdot\|\right) \\
& =\left\{\left(x_{k}\right) \in w(n-X):\left\{n \in \mathbb{N}: \frac{1}{n} \sum_{k=1}^{n}\left[M\left(\left\|\frac{\Delta_{m}^{(s)} x_{k}-l}{\rho}, z_{1}, z_{2} \ldots z_{n-1}\right\|\right)\right]^{p_{k}} \geq \varepsilon\right\} \in I,\right. \\
& \left.\quad \text { for some } \rho>0, l \in X \text { and } \forall z_{1}, z_{2} \ldots z_{n-1} \in X\right\}, \\
& W_{0}^{I}\left(\Delta_{m}^{(s)}, M, p,\|\cdot \ldots, \cdot\|\right) \\
& =\left\{\left(x_{k}\right) \in w(n-X):\left\{n \in \mathbb{N}: \frac{1}{n} \sum_{k=1}^{n}\left[M\left(\left\|\frac{\Delta_{m}^{(s)} x_{k}}{\rho}, z_{1}, z_{2} \ldots . z_{n-1}\right\|\right)\right]^{p_{k}} \geq \varepsilon\right\} \in I,\right. \\
& \left.\quad \text { for some } \rho>0, l \in X \text { and } \forall z_{1}, z_{2} \ldots z_{n-1} \in X\right\}, \\
& W_{\infty}\left(\Delta_{m}^{(s)}, M, p,\|\cdot, \ldots, \cdot\|\right) \\
& =\left\{\left(x_{k}\right) \in w(n-X): \sup _{n \in \mathbb{N}} \frac{1}{n} \sum_{k=1}^{n}\left[M\left(\left\|\frac{\Delta_{m}^{(s)} x_{k}}{\rho}, z_{1} z_{2}, \ldots, z_{n-1}\right\|\right)\right]^{p_{k}}<\infty,\right.
\end{aligned}
$$$$
\text { for some } \left.\rho>0, l \in X \text { and } \forall z_{1}, z_{2} \ldots z_{n-1} \in X\right\} \text {, }
$$

$W_{\infty}^{I}\left(\Delta_{m}^{(s)}, M, p,\|\cdot \ldots, \cdot\|\right)$

$$
\begin{aligned}
& =\left\{\left(x_{k}\right) \in w(n-X): \exists K>0 \text { s.t. }\left\{n \in \mathbb{N}: \frac{1}{n} \sum_{k=1}^{n}\left[M\left(\left\|\frac{\Delta_{m}^{(s)} x_{k}}{\rho}, z_{1} z_{2}, \ldots, z_{n-1}\right\|\right)\right]^{p_{k}} \geq K\right\} \in I,\right. \\
& \text { for some } \left.\rho>0, l \in X \text { and } \forall z_{1}, z_{2} \ldots z_{n-1} \in X\right\},
\end{aligned}
$$

Note 2. For $s=0$, we write the above spaces as $Z(M, p,\|\cdot, \ldots, \cdot\|)$, where $Z=W^{I}, W_{0}^{I}$, $W_{\infty}$, and $W_{\infty}^{I}$.

It is clear from definitions that

$$
W_{0}^{I}\left(\Delta_{m}^{(s)}, M, p,\|\cdot, \ldots, \cdot\|\right) \subset W^{I}\left(\Delta_{m}^{(s)}, M, p,\|\cdot, \ldots, \cdot\|\right) \subset W_{\infty}\left(\Delta_{m}^{(s)}, M, p,\|\cdot, \ldots, \cdot\|\right)
$$


Corollary 3.9. The sequence spaces $Z\left(\Delta_{m}^{(s)}, M, p,\|\cdot, \ldots, \cdot\|\right)$ are paranormed spaces (not totally paranormed) with respect to the paranormed $h_{\Delta}$ defined by

$$
\begin{gathered}
h_{\Delta}(x)=\inf \left\{\rho^{p_{k} / H}: \sup _{k} M\left(\left\|\frac{\Delta_{m}^{(s)} x_{k}}{\rho}, z_{1}, z_{2}, \ldots, z_{n-1}\right\|\right) \leq 1,\right. \\
\text { for some } \left.\rho>0 \text { and } \forall z_{1}, z_{2}, \ldots, z_{n-1} \in X\right\},
\end{gathered}
$$

where $H=\max \left\{1, \sup p_{k}\right\}$ and $Z=W^{I}, W_{0}^{I}, W_{\infty}$, and $W_{\infty}^{I}$.

Remark 3.10. It is obvious that $\left(x_{k}\right) \in Z\left(\Delta_{m}^{s}, M, p,\|\cdot, \ldots, \cdot\|\right)$ if and only if $Z\left(\Delta_{m}^{(s)}, M, p\right.$, $\|\cdot, \ldots, \cdot\|)$, for $Z=W^{I}, W_{0}^{I}, W_{\infty}^{I}$ and $W_{\infty}$.

Also it is clear that the paranorm $g_{\Delta}$ and $h_{\Delta}$ are equivalent.

We state the following Theorem in view of the Lemma 2.17.

Theorem 3.11. Let $X$ be a standard n-normed space and $\left\{e_{1}, e_{2}, \ldots, e_{n}\right\}$ an orthogonal set in $X$. Then, the following hold:

(a) $W^{I}\left(\Delta_{m}^{s}, M, p,\|\cdot, \ldots, \cdot\|_{\infty}\right)=W^{I}\left(\Delta_{m}^{s}, M, p,\|\cdot, \ldots, \cdot\|_{(n-1)}\right)$,

(b) $W_{0}^{I}\left(\Delta_{m}^{s}, M, p,\|\cdot, \ldots, \cdot\|_{\infty}\right)=W_{0}^{I}\left(\Delta_{m}^{s}, M, p,\|\cdot, \ldots, \cdot\|_{(n-1)}\right)$,

(c) $W_{\infty}\left(\Delta_{m}^{s}, M, p,\|\cdot, \ldots, \cdot\|_{\infty}\right)=W_{\infty}\left(\Delta_{m}^{s}, M, p,\|\cdot \ldots, \cdot\|_{(n-1)}\right)$,

(d) $W_{\infty}^{I}\left(\Delta_{m}^{s}, M, p,\|\cdot, \ldots, \cdot\|_{\infty}\right)=W_{\infty}^{I}\left(\Delta_{m}^{s}, M, p,\|\cdot, \ldots, \cdot\|_{(n-1)}\right)$, where $\|\cdot, \ldots, \cdot\|_{\infty}$ is the derived $(n-1)$-norm defined with respect to the set $\left\{e_{1}, e_{2}, \ldots, e_{n}\right\}$ and $\|\cdot, \ldots, \cdot\|_{(n-1)}$ is the standard $(n-1)$-norm on $X$.

Remark 3.12. Theorem 3.11 also holds if we replace the difference operator $\Delta_{m}^{s}$ by the difference operator $\Delta_{m}^{(s)}$.

Theorem 3.13. The spaces $Z\left(\Delta_{m}^{(s)}, M, p,\|\cdot, \ldots, \cdot\|\right)$ and $Z(M, p,\|\cdot, \ldots, \cdot\|)$ are equivalent as topological spaces, where $Z=W^{I}, W_{0}^{I}, W_{\infty}^{I}$, and $W_{\infty}$.

Proof. Consider the mapping $T: Z\left(\Delta_{m}^{(s)}, M, p,\|\cdot, \ldots, \cdot\|\right) \rightarrow Z(M, p,\|\cdot, \ldots, \cdot\|)$ defined by

$$
T x=\left(\Delta^{(s)} x_{k}\right), \quad \text { for each } x=\left(x_{k}\right) \in Z\left(\Delta_{m}^{(s)}, M, p,\|\cdot, \ldots, \cdot\|\right) .
$$

Then, clearly, $T$ is a linear homeomorphism and the proof follows.

\section{References}

[1] H. Fast, "Sur la convergence statistique," Colloquium Mathematicum, vol. 2, pp. 241-244, 1951.

[2] I. J. Schoenberg, "The integrability of certain functions and related summability methods," The American Mathematical Monthly, vol. 66, no. 5, pp. 361-375, 1959.

[3] J. A. Fridy, “On statistical convergence," Analysis, vol. 5, no. 4, pp. 301-313, 1985.

[4] P. Kostyrko, T. Šalàt, and W. Wilczyński, "On I-convergence," Real Analysis Exchange, vol. 26, no. 2, pp. 669-685, 2000-2001. 
[5] H. Kizmaz, "On certain sequence spaces," Canadian Mathematical Bulletin, vol. 24, no. 2, pp. 169-176, 1981.

[6] M. Et and R. Çolak, "On generalized difference sequence spaces," Soochow Journal of Mathematics, vol. 21, no. 4, pp. 147-169, 1985.

[7] B. C. Tripathy and A. Esi, "A new type of sequence spaces," International Journal of Environmental Science and Technology, vol. 1, no. 1, pp. 11-14, 2006.

[8] B. C. Tripathy, A. Esi, and B. K. Tripathy, "On a new type of generalized difference Cesàro sequence spaces," Soochow Journal of Mathematics, vol. 31, no. 3, pp. 333-340, 2005.

[9] S. Gähler, "Linear 2-normietre Räume," Mathematische Nachrichten, vol. 28, pp. 1-43, 1965.

[10] A. Misiak, "n-inner product spaces," Mathematische Nachrichten, vol. 140, no. 1, pp. 299-319, 1989.

[11] H. Gunawan, "The spaces of $p$-summable sequences and its natural n-norm," Bulletin of the Australian Mathematical Society, vol. 64, no. 1, pp. 137-147, 2001.

[12] H. Gunawan and M. Mashadi, "On n-normed spaces," International Journal of Mathematics and Mathematical Sciences, vol. 27, no. 10, pp. 631-639, 2001.

[13] H. Gunawan and M. Mashadi, "On finite-dimensional 2-normed spaces," Soochow Journal of Mathematics, vol. 27, no. 3, pp. 147-169, 2001.

[14] M. Gungor and M. Et, " $\Delta^{r}$-strongly almost summable sequences defined by Orlicz functions," Indian Journal of Pure and Applied Mathematics, vol. 34, no. 8, pp. 1141-1151, 2003.

[15] E. Savas, " $\Delta^{m}$-strongly summable sequences spaces in 2-normed spaces defined by ideal convergence and an Orlicz function," Applied Mathematics and Computation, vol. 217, no. 1, pp. 271-276, 2010.

[16] M. Gürdal, "On ideal convergent sequences in 2-normed spaces," Thai Journal of Mathematics, vol. 4, no. 1, pp. 85-91, 2006.

[17] M. Gürdal and A. Sahiner, "Ideal convergence in n-normal spaces and some new sequence spaces via n-norm," Journal of Fundamental Sciences, vol. 4, no. 1, pp. 233-244, 2008.

[18] I. J. Maddox, "Sequence spaces defined by a modulus," Mathematical Proceedings of the Cambridge Philosophical Society, vol. 100, no. 1, pp. 161-166, 1986.

[19] J. Lindenstrauss and L. Tzafriri, "On Orlicz sequence spaces," Israel Journal of Mathematics, vol. 10, pp. 379-390, 1971.

[20] S. D. Parashar and B. Choudhary, "Sequence spaces defined by Orlicz functions," Indian Journal of Pure and Applied Mathematics, vol. 25, no. 4, pp. 419-428, 1994.

[21] M. Et, Y. Altin, B. Choudhary, and B. C. Tripathy, “On some classes of sequences defined by sequences of Orlicz functions," Mathematical Inequalities E Applications, vol. 9, no. 2, pp. 335-342, 2006.

[22] A. Şahiner and M. Gürdal, "New sequence spaces in $n$-normed spaces with respect to an Orlicz function," The Aligarh Bulletin of Mathematics, vol. 27, no. 1, pp. 53-58, 2008.

[23] B. C. Tripathy, M. ET, and Y. Altin, "Generalized difference sequence spaces defined by Orlicz function in a locally convex space," Journal of Analysis and Applications, vol. 3, no. 1, pp. 175-192, 2003.

[24] P. K. Kamthan and M. Gupta, Sequence Spaces and Series, Lecture Notes in Pure \& Applied Mathematics, Marcel Dekker, New York, NY, USA, 1980. 


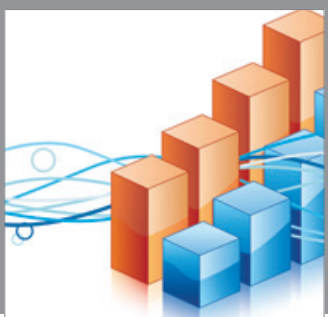

Advances in

Operations Research

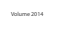

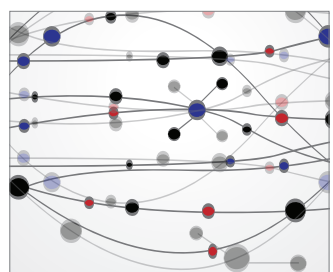

\section{The Scientific} World Journal
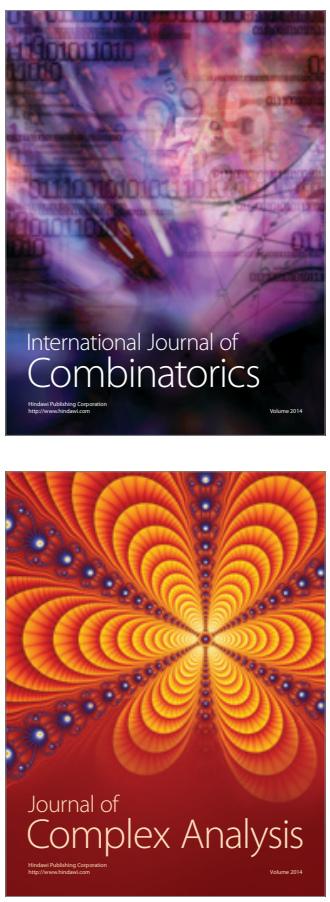

International Journal of

Mathematics and

Mathematical

Sciences
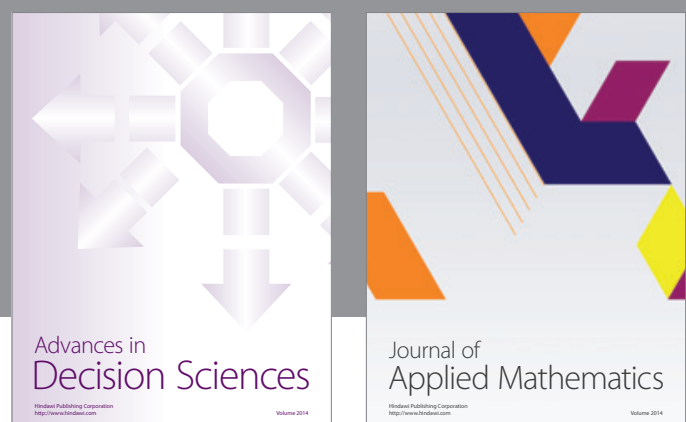

Journal of

Applied Mathematics
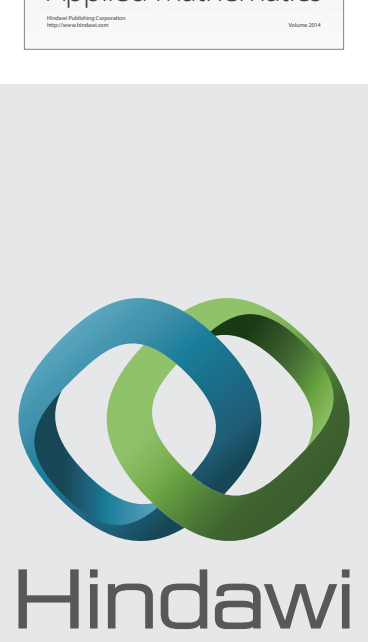

Submit your manuscripts at http://www.hindawi.com
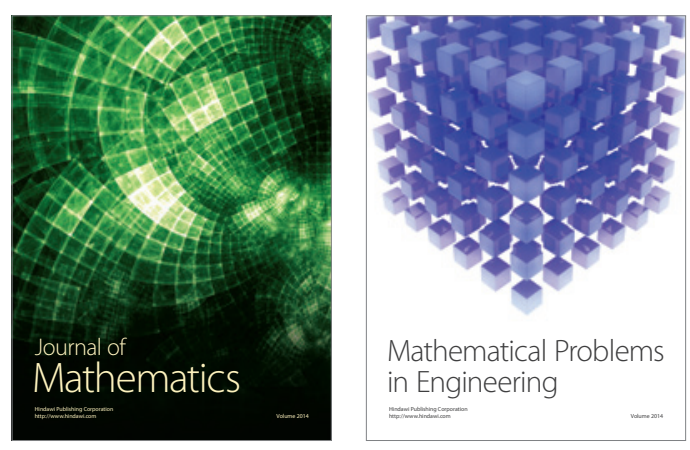

Mathematical Problems in Engineering
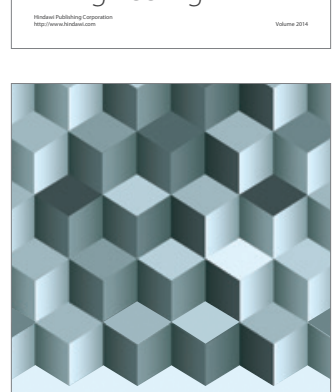

Journal of

Function Spaces
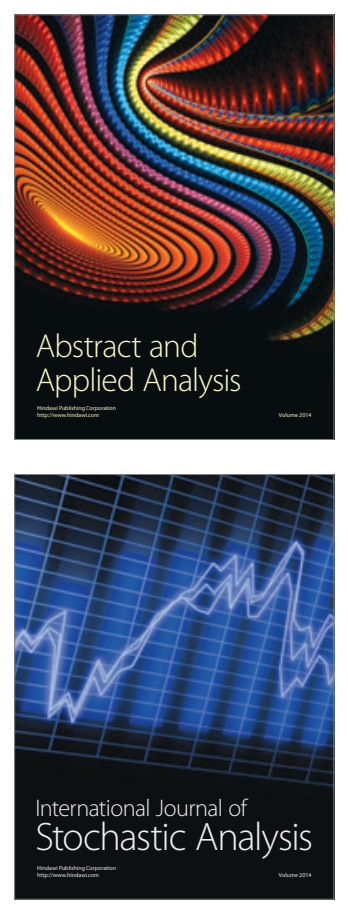

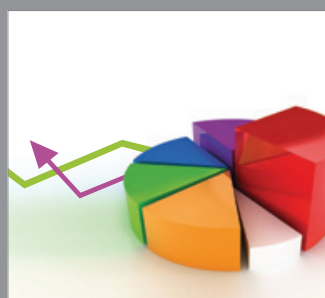

ournal of

Probability and Statistics

Promensencen
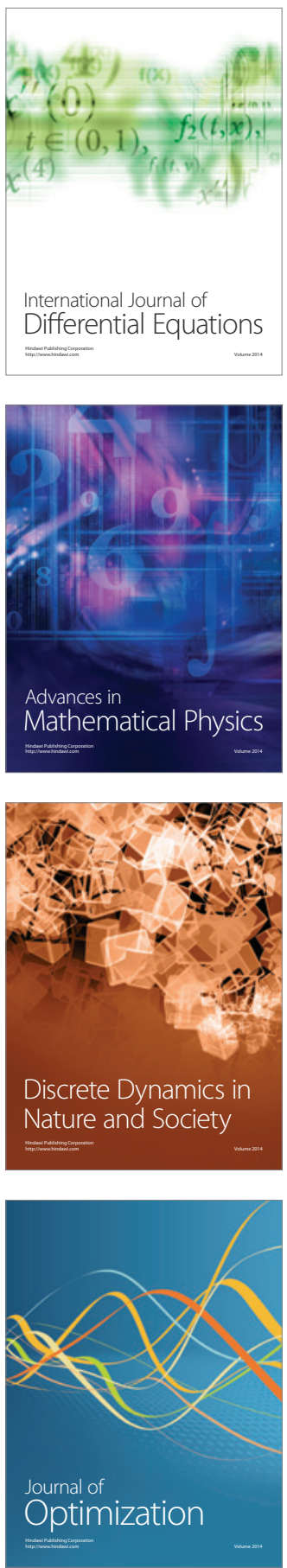